\title{
Clinical study on collagen gel droplet-embedded culture drug sensitivity test for multidrug combination chemotherapy and super selective intra-arterial infusion chemoradiotherapy in oral squamous cell carcinoma
}

\author{
KANAME SAKUMA ${ }^{1}$, RYUKI TAMURA ${ }^{2}$, SHINTARO HANYU ${ }^{2}$, HARUKA TAKAHASHI ${ }^{2}$, \\ HIDEAKI SATO $^{1}$, TAKAHIRO ONEYAMA ${ }^{1}$, AKIRA YAMAGUCHI ${ }^{1}$ and AKIRA TANAKA ${ }^{2}$ \\ ${ }^{1}$ Department of Oral and Maxillofacial Surgery, Niigata Hospital, The Nippon Dental University, \\ Niigata, Niigata 951-8580; ${ }^{2}$ Department of Oral and Maxillofacial Surgery, The Nippon Dental \\ University School of Life Dentistry at Niigata, Niigata, Niigata 951-8580, Japan
}

Received July 13, 2017; Accepted October 17, 2017

DOI: $10.3892 /$ mco.2017.1459

\begin{abstract}
Using trace three-dimensional culture, the collagen gel droplet-embedded culture drug sensitivity test (CD-DST) can be tested even in cases with a small number of cells, including oral squamous cell carcinoma (OSCC), and evaluation of the antitumor effect with a drug concentration close to the in vivo level is possible. The present report aimed to evaluate the utility of the CD-DST in the assessment of the in vitro efficacy of single-agent and multidrug combination chemotherapy for OSCC in comparison with the clinical response rates and to examine the possible clinical application of CD-DST for such cases. A total of 33 OSCC patients from whom 33 samples were obtained from January 2010 to September 2015 were included. CD-DST was performed, individually and in combination, on the three drugs [i.e., cisplatin (CDDP), 5-fluorouracil (5-FU), and docetaxel (DOC)] and on super selective intra-arterial infusion chemoradiotherapy (IACRT). The overall evaluable rate of the CD-DST in OSCC was $81.8 \%$ (27 of 33 cases) and the sensitivity to each anticancer drug was evaluated. The in vitro efficacy rates of IACRT, cisplatin +5 -fluorouracil, and docetaxel + cisplatin +5 -fluorouracil (TPF) confirmed the estimated clinical response rates. In 14 of 33 patients, the results of CD-DST were compared with clinical efficacy, which was judged based on measurable lesions on imaging. For TPF therapy, the sensitivity test of the IACRT had a positive
\end{abstract}

Correspondence to: Dr Kaname Sakuma, Department of Oral and Maxillofacial Surgery, Niigata Hospital, The Nippon Dental University, 1-8 Hamaura-cho, Chuo-ku, Niigata, Niigata 951-8580, Japan

E-mail: sakuma.k@ngt.ndu.ac.jp

Key words: anticancer drug sensitivity test, oral squamous cell carcinoma, collagen gel droplet-embedded culture drug sensitivity test, multidrug combination chemotherapy, super selective intraarterial chemoradiotherapy predictive value of $90.9 \%$ (10 of 11 cases) and a negative predictive value of $100 \%$ ( 3 of 3 cases); the accuracy of the susceptibility test for the anticancer agents was $92.8 \%$ (13 of 14 cases). The CD-DST may be useful in selecting multidrug combination chemotherapy and IACRT for OSCC, however, accumulation of further clinical data is required in the future.

\section{Introduction}

The treatment of advanced or unresectable oral squamous cell carcinoma (OSCC) is multidisciplinary and entails the use of both multidrug combination chemotherapy and radiotherapy (1-3). The usefulness of super selective intraarterial infusion chemoradiotherapy (IACRT) to deliver high concentrations of anticancer drugs to tumors has been reported (4). The selection of such anticancer drugs for IACRT had been based on statistical information and clinical reports on a number of cases (1-3); however, the expected therapeutic effects of these anticancer drugs have not been consistent and adverse events were common. Therefore, drug sensitivity testing prior to administration of an anticancer agent is ideal to avoid the serious side effects of less effective anticancer drugs. Collagen gel droplet-embedded culture drug sensitivity test (CD-DST) uses an image colorimetric method to assess the combination of an anticancer drug and microcollagen gel embedded in a three-dimensional serum-free culture medium (3 to $10 \times 10^{3}$ cells $/ 30 \mu \mathrm{g} / \mathrm{drop}$ ) $(5,6)$. It is a susceptibility test that is currently widely applied in various clinical fields, including gastrointestinal cancer (7-9). However, reports on the use of CD-DST in OSCC were few and none have evaluated multidrug combination chemotherapy or IACRT.

CD-DST is usually carried out on surgical and biopsy specimens. In this study, we aimed to evaluate the utility of the CD-DST in the assessment of the in vitro efficacy of single-agent and multidrug combination chemotherapy for OSCC in comparison with the clinical response rates and to examine the possible clinical application of the CD-DST for such cases. 


\section{Patients and methods}

The subjects comprised 33 OSCC patients who gave consent to participate in the study from January 2010 to September 2015 at Nippon Dental University Niigata Hospital Oral Maxillofacial Surgery (Table I). The Ethics Committee of The Nippon Dental University School of Life Dentistry at Niigata approved the present study (approval no. ECNG-H-119).

CD-DST was performed on 31 primary lesions and 2 metastatic lymph nodes; 5-7- $\mathrm{mm}^{2}$ tissues (about 0.25-0.5 mg) were collected mainly from the area surrounding the hardened part of the tumor. CD-DST was carried out according to the method invented and reported by Kobayashi et al $(5,6)$, who used a human tumor cell primary culture system kit (Primaster ${ }^{\circledR}$; Kurabo Industries Ltd., Osaka, Japan). Briefly, each sample was washed five times with $50 \mathrm{ml}$ saline containing $1.0 \mathrm{mg} / \mathrm{ml}$ penicillin, $0.5 \mathrm{mg} / \mathrm{ml}$ kanamycin, and $2.5 \mu \mathrm{g} / \mathrm{ml}$ amphotericin B; this was followed by treatment with Dispersion Enzyme Cocktail EZ (Primaster ${ }^{\circledR}$ reagent). The obtained cell suspension samples were inoculated into collagen gel-coated flasks (CG flasks, a Primaster ${ }^{\circledR}$ device) and were inoculated overnight in a pre-culture medium PCM-1 (Primaster ${ }^{\circledR}$ content) at $37^{\circ} \mathrm{C}$ in $5 \%$ $\mathrm{CO}_{2}$. Next, the collagen gel was digested with $0.05 \% \mathrm{EZ}$ and viable cancer cells were obtained. Type I collagen, F-12 medium that was 10 times concentrated, and reconstitution buffer were mixed together in ice water at a ratio of 8:1:1 (Primaster ${ }^{\circledR}$ content). The prepared cancer cell suspension was added to the collagen solution at a final density of $1 \times 105$ cells $/ \mathrm{ml}$. Three drops of the collagen-cell mixture (30 $\mu \mathrm{l} / \mathrm{drop})$ were placed in a 6-well plate on ice and was allowed to solidify at $37^{\circ} \mathrm{C}$ in a $\mathrm{CO}_{2}$ incubator; the final concentration was about $3 \times 10^{3}$ cells per collagen gel droplet. One hour later, a DF medium containing $10 \%$ fetal bovine serum was overlaid in each well; the plates were placed in $\mathrm{CO}_{2}$ incubator overnight at $37^{\circ} \mathrm{C}$. The anticancer drugs were added with the following final concentrations and incubated for $24 \mathrm{~h}: 0.5 \mu \mathrm{g} / \mathrm{ml}$ cisplatin (CDDP) (10), $0.7 \mu \mathrm{g} / \mathrm{ml}$ fluorouracil (5-FU) (10), and $0.1 \mu \mathrm{g} / \mathrm{ml}$ docetaxel (DOC) (11). For cases that needed multidrug combination chemotherapy, the respective concentrations were placed together in the same well. The duration of CDDP contact, even at a concentration $2.5 \mu \mathrm{g} / \mathrm{ml}$ (CDDP extreme contact) for IACRT, was the same at $24 \mathrm{~h}$. The concentration of each anticancer drug in the culture medium was determined so as to exhibit area under the curve values that were similar to those in serum during the first $24 \mathrm{~h}$ of intravenous administration of the corresponding drug at the standard clinical dosage. After removal of the medium containing the anticancer drugs, each well was rinsed twice with $3 \mathrm{ml}$ of Hanks' balanced salt solution, overlaid with $4 \mathrm{ml}$ of PCM-2 medium (Primaster ${ }^{\circledR}$ serum-free medium), and incubated for seven more days. At the end of the incubation, a neutral red solution was added to each well to make a final concentration of $50 \mu \mathrm{g} / \mathrm{ml}$; colonies in the collagen gel droplets were stained for two h. Each collagen droplet was fixed with $10 \%$ neutral buffered formalin, washed in water, air dried, and quantified by optical density image analysis using the Primage System (Solution Systems, Tokyo, Japan).

In vitro sensitivity was expressed as the $\mathrm{T} / \mathrm{C}$ ratio of the optical density, where $\mathrm{T}$ represented the treated samples and $\mathrm{C}$ represented the controls; a $\mathrm{T} / \mathrm{C}$ ratio of $<50 \%$ was regarded as chemosensitive in vitro. A tumor cell colony volume ratio
Table I. Characteristics of patients $(\mathrm{N}=33)$.

Number of patients (\%)

Sex

Male

$22(66.6)$

Female

$11(33.3)$

Age, years

Mean

$74(37-95)$

Histology (Squamous cell carcinoma)

Differentiation

Well-differentiated

Moderately-differentiated

Poorly-differentiated

$3(9.0)$

Stage

I

II

$12(36.3)$

$6(18.1)$

IV

$13(39.3)$

Primary site

Tongue

Buccal mucosa

Oral floor

Gingiva

Hard palate

Resection mode

Biopsy

Surgery

Sample site

Neck metastasis

Primary

$31(93.9)$

Others (Neck metastasis)

2 (6.0)

CD-DST

Success

27 (81.8)

Failure

$6(18.2)$

CD-DST, collagen gel droplet-embedded culture drug sensitivity test.

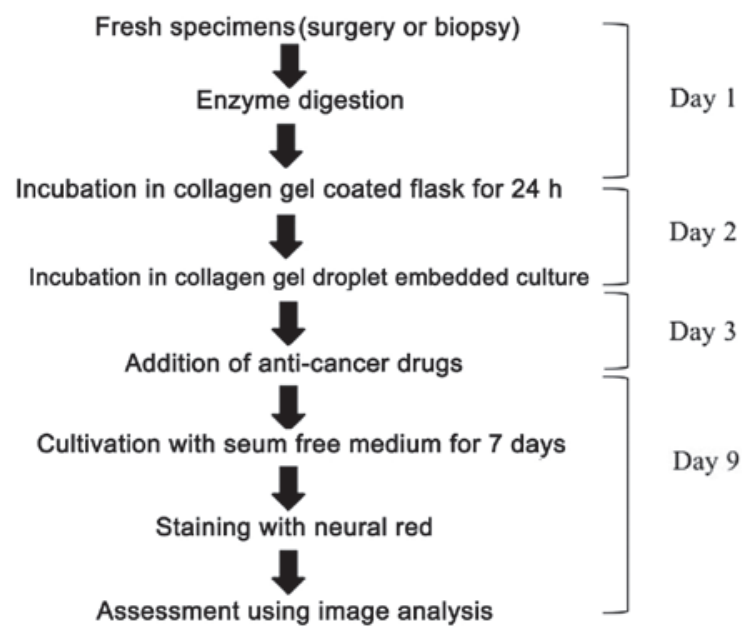

Figure 1. Overview of the collagen gel droplet-embedded culture drug sensitivity test method. 
Table II. Evaluable rate of the clinical specimens of OSCC on CD-DST.

\begin{tabular}{lccc}
\hline & Primary lesion & Metastatic lymph node & Total \\
\hline Number of samples & 31 & 2 & 33 \\
Evaluable cases & 26 & 1 & 27 \\
Evaluable rate $(\%)$ & $83.8(26 / 31)^{\mathrm{a}}$ & $50.0(1 / 2)$ & $81.8(27 / 33)$ \\
\hline
\end{tabular}

${ }^{\mathrm{a} N u m b e r}$ of success cases/total number of assays.

Table III. In vitro sensitivity and clinical efficacy rates of the individual drugs for OSCC

\begin{tabular}{lcccccc}
\hline & CDDP & 5-FU & DOC & TPF & PF & IACRT \\
\hline CD-DST in vitro & 23.8 & 30.0 & 37.5 & 80.7 & 22.2 & 87.5 \\
Efficacy rate (\%) & $(5 / 21)^{\mathrm{a}}$ & $(3 / 10)$ & $(3 / 8)$ & $(21 / 26)$ & $(4 / 18)$ & $(14 / 16)$ \\
Clinical efficacy rate (\%) & $26.3(13)$ & $13.0(14)$ & $10.0(15)$ & $80.6(16)$ & $20.0-30.0(17,18)$ & $85.0-91.2(19,20)^{\mathrm{b}}$ \\
\hline
\end{tabular}

In vitro drug sensitivity was defined as positive when the $\mathrm{T} / \mathrm{C}$ ratio was $\leq 50 \%$. CD-DST, collagen gel droplet-embedded culture drug sensitivity test; CDDP, cisplatin; 5-FU, 5-fluorouracil; DOC, docetaxel; TPF, DOC+CDDP+5-FU; PF, CDDP+5-FU; IACRT, super selective intraarterial infusion chemoradiotherapy. ${ }^{a}$ Number of effective cases/total number of assays. ${ }^{b}$ Evaluated with complete response only.

(tumor growth rate) at 0 time was calculated from the control group; a value of less than 0.8 was regarded as unsuccessful culture (low growth rate), regardless of the tumor cell colony volume of the control group.

Retrospective comparison between clinical response, as measured by tumor shrinkage and the results of drug sensitivity on CD-DST was conducted on 14 cases (42.4\%) that had measurable lesions on imaging. We studied five cases of TPF therapy and nine cases of IACRT; TPF therapy was intravenously administered with DOC: $50 \mathrm{mg} / \mathrm{m}^{2}$, CDDP: $50 \mathrm{mg} / \mathrm{m}^{2}$, 5-FU: $2,500 \mathrm{mg} / \mathrm{m}^{2}$. The latter was administered weekly using CDDP at a cumulative dose of $80-460 \mathrm{mg} /$ body (median, $350 \mathrm{mg} /$ body) and radiation therapy was given at 2 Gy/day at a cumulative dose of 56-72 Gy (median, 66 Gy). Response was evaluated in accordance with the Response Evaluation Criteria in Solid Tumors (12). Partial response (PR) and complete response $(\mathrm{CR})$ were judged to be sensitive.

Fisher's exact test was used to determine differences between groups. A value of $\mathrm{P}<0.05$ was considered statistically significant (SPSS, Inc., Chicago, IL, USA).

\section{Results}

Evaluable rate of clinical specimens. CD-DST in OSCC had an overall evaluable rate of $81.8 \%$ (27 of 33 cases) and could be applied to each of the anticancer agent in this study. The evaluable rate was $83.8 \%$ (26 of 31 cases) for primary lesions and $50 \%$ ( 1 of 2 cases) for metastatic lymph nodes (Table II). Causes of unsuccessful CD-DST were insufficient tumor cells (growth rate less than 0.8 ) in three cases and bacterial contamination in three cases. The mean tumor growth rate was $10.1 \pm 4.92$.

Comparison between in vitro effective rate and clinical response rate of various anticancer drugs. Results of the comparison between the in vitro effective rates and clinical response rates of representative anticancer drugs for clinical treatment of OSCC (13-20) are shown in Table III. 5-FU and DOC had slightly higher clinical response rates than in vitro effective rates, but CDDP had comparable clinical response and in vitro effective rates. The in vitro effective rates and clinical response rates were comparable in docetaxel + cisplatin + 5-fluorouracil (TPF) and cisplatin + 5-fluorouracil (PF) therapies, as well as in CDDP at high concentrations mimicking IACRT. In addition, no significant difference was observed among the susceptibilities of various anticancer agents, regardless of stage and histologic differentiation $(\mathrm{P}>0.05)$.

Comparison between clinical response and anticancer drug sensitivity test. The clinical response of 14 patients with measurable lesions by imaging diagnosis is shown in Table IV. Among patients who received TPF therapy, three had PR and two had stable disease (SD). For those who received IACRT, eight cases had CR and one case had progressive disease (PD). There were three cases of TPF therapy and seven cases of IACRT that were determined by the CD-DST to be highly sensitive. The corresponding drug sensitivity test results of the 14 are shown in Table V. Among the cases that received TPF therapy and IACRT, all three cases with PR and seven of eight cases with CR were highly sensitive; all cases with SD and PD had low sensitivity and agreed with the CD-DST. In the $88.8 \%$ (8 of 9 cases) who received IACRT, the results of the CD-DST performed at a high CDDP contact concentration revealed were correlated with the clinical results. In addition, in the $100 \%$ (5 of 5 cases) who received TPF, the results of the CD-DST performed at a TPF contact revealed were correlated with the clinical results. TPF therapy and IACRT had a positive predictive value of $90.9 \%$ (10 of 11 cases) and a negative 
Table IV. Association between in vitro sensitivity rate and chemotherapeutic effect.

\begin{tabular}{|c|c|c|c|c|c|c|c|}
\hline & Evaluated lesion & $\begin{array}{l}\text { Treatment } \\
\text { regimen }\end{array}$ & $\begin{array}{l}\text { Clinical } \\
\text { effect }\end{array}$ & Sensitivity test & $\begin{array}{c}\mathrm{T} / \mathrm{C} \text { value } \\
(\%)\end{array}$ & $\begin{array}{l}\text { Growth } \\
\text { rate }\end{array}$ & $\begin{array}{c}\text { CD-DST/clinical } \\
\text { effect }\end{array}$ \\
\hline 1 & Gingiva & TPF & PR & $\mathrm{TPF}$ & 22.8 & 5.8 & $\mathrm{~S} / \mathrm{S}$ \\
\hline 2 & Tongue & $\mathrm{TPF}$ & PR & $\mathrm{TPF}$ & 27.7 & 15.03 & $\mathrm{~S} / \mathrm{S}$ \\
\hline 3 & Buccal mucosa & TPF & SD & $\mathrm{TPF}$ & 54.2 & 7.96 & $\mathrm{R} / \mathrm{R}$ \\
\hline 4 & Hard palate & TPF & SD & $\mathrm{TPF}$ & 71.6 & 2.59 & $\mathrm{R} / \mathrm{R}$ \\
\hline 5 & Tongue (metastatic lymph node) & TPF & PR & $\mathrm{TPF}$ & 32.2 & 1.55 & $\mathrm{~S} / \mathrm{S}$ \\
\hline 6 & Tongue & IACRT & $\mathrm{CR}$ & CDDP (extreme) & 19.4 & 11.22 & $\mathrm{~S} / \mathrm{S}$ \\
\hline 7 & Gingiva & IACRT & $\mathrm{CR}$ & CDDP (extreme) & 31.2 & 2.89 & $\mathrm{~S} / \mathrm{S}$ \\
\hline 8 & Tongue & IACRT & $\mathrm{CR}$ & CDDP (extreme) & 42.3 & 17.65 & $\mathrm{~S} / \mathrm{S}$ \\
\hline 9 & Gingiva & IACRT & $\mathrm{CR}$ & CDDP (extreme) & 42.6 & 3.88 & $\mathrm{~S} / \mathrm{S}$ \\
\hline 10 & Gingiva & IACRT & PD & CDDP (extreme) & 62.3 & 15.4 & $\mathrm{R} / \mathrm{R}$ \\
\hline 11 & Tongue & IACRT & $\mathrm{CR}$ & CDDP (extreme) & 11.6 & 9.13 & $\mathrm{~S} / \mathrm{S}$ \\
\hline 12 & Buccal mucosa & IACRT & $\mathrm{CR}$ & CDDP (extreme) & 22.7 & 2.86 & $\mathrm{~S} / \mathrm{S}$ \\
\hline 13 & Tongue & IACRT & $\mathrm{CR}$ & CDDP (extreme) & 34.0 & 1.39 & $\mathrm{~S} / \mathrm{S}$ \\
\hline 14 & Gingiva & IACRT & $\mathrm{CR}$ & CDDP (extreme) & 118.0 & 0.87 & $\mathrm{R} / \mathrm{S}$ \\
\hline
\end{tabular}

In vitro drug sensitivity was defined as sensitive when the $\mathrm{T} / \mathrm{C}$ rate was $\leq 50 \%$. CD-DST, collagen gel droplet-embedded culture drug sensitivity test; T/C, total colony of the treated cells/total colony of the untreated cells; CR, complete response; PR, partial response; SD, stable disease; PD, progressive disease; CDDP, cisplatin; 5-FU, 5-fluorouracil; DOC, docetaxel; TPF, DOC+CDDP+5-FU; IACRT, super selective intraarterial infusion chemoradiotherapy; $\mathrm{S}$, sensitive; $\mathrm{R}$, resistant.

Table V. Summary of the association between in vitro sensitivity data and chemotherapeutic effect.

\begin{tabular}{lllll}
\hline & \multicolumn{4}{c}{ Chemotherapeutic effect } \\
\cline { 2 - 5 } & CR & PR & SD & PD \\
\hline In vitro chemosensitivity data & & & & \\
High sensitivity & 7 & 3 & 0 & 0 \\
Low sensitivity (resistant) & 1 & 0 & 2 & 1
\end{tabular}

CR, complete response; PR, partial response; $\mathrm{SD}$, stable disease; PD, progressive disease.

predictive value of $100 \%$ (3 of 3 cases). The diagnostic accuracy was $92.8 \%$ (13 of 14 cases).

\section{Discussion}

The standard chemotherapy in the head and neck region that were reported to exert beneficial effects comprises PF therapy, which is a combination of CDDP and a 5-FU-type pyrimidine antimetabolite $(17,18)$, and TPF therapy, which is a combination of CDDP, 5-FU, and a taxane type of anticancer drug (1-3). However, TPF therapy had been reported to have high rates of adverse reactions, including grade 3 or higher neutropenia at 76.9 to $83.0 \%$, febrile neutropenia at 5.2 to $12.0 \%$, and severe hematologic toxicity, in addition to mucositis, nausea, and vomiting $(21,22)$. From this point of view, the clinical application of in vitro chemosensitivity test is a promising strategy.
There have been previously published in vitro methods of anticancer drug sensitivity test, such as the human tumor clonogenic assay and the succinate dehydrogenase inhibition test; however, the success rate of these two-dimensional primary culture methods have been limited by drug contact concentration $(23,24)$. On the other hand, the CD-DST that we performed in this study had a potential for a high success rate on initial culture, needed a small number of cells, and used serum-free culture medium and image colorimetry to eliminate the influence of fibroblasts. The CD-DST has the advantage of using an anticancer drug contact concentration that is comparable to the clinical therapeutic dose $(5,6,10,25)$. In fact, the primary measurement success rate of the CD-DST was reported to be $87.5 \%$ in colorectal cancer, $79.2 \%$ in lung cancer, and $84.3 \%$ in breast cancer $(11,26,27)$. For OSCC, the primary culture rate was $83.8 \%$ (26 of 31 cases) for primary tumors, $50 \%$ ( 1 of 2 cases) for metastatic lymph nodes, and $81.8 \%$ (27 of 33 cases) overall.

Notably, CD-DST was clinically applicable even with the use of small tissue specimens of OSCC. For cases that could not be evaluated by the CD-DST, the lack of cells may have been due to biopsy technique. Yamamoto et al (28) reported that in breast cancer, sampling should be performed mainly from the central part of the tumor. In the non-evaluable cases in this study, collection of OSCC samples may have been from the superficial necrotic portions of the tumor. Therefore, it is important to collect tumor specimens from the hardened portions during biopsy.

Kobayashi et al $(5,6)$ reported a statistically significant correlation between in vitro efficacy on CD-DST and clinical response in breast cancer, gastric cancer, colorectal cancer, and lung cancer. In this study on OSCC, the $80.7 \%$ in vitro 
efficacy rate of TPF therapy was comparable to the previously reported $80.6 \%$ clinical response rate to preoperative TPF chemotherapy (16). Furthermore, the $22.2 \%$ in vitro efficacy rate of PF therapy in this study was comparable to the reported clinical response rate of 20 to $30 \%(17,18)$. For multidrug combination therapy, it was suggested that the in vitro effective rate on CD-DST reproduced the clinical response rate. Drug sensitivity test of IACRT has not been reported; as a substitute and to reproduce the clinical response rate of IACRT in combination with radiation therapy, a high concentration contact of CDDP was used. As a result, the $87.5 \%$ in vitro efficacy rate of IACRT was comparable to the previously reported 85 to $91.2 \%(19,20)$. In the $88.8 \%$ (8 of 9 cases) who received IACRT, the results of the CD-DST performed at a high CDDP contact concentration revealed were correlated with the clinical results. Our results suggested that a high contact concentration of CDDP can be applied to represent CD-DST of IACRT in OSCC.

In one patient (case 14), the result of the susceptibility test and clinical effect were different. Nakagawa et al (29) reported that if the growth rate was low, the sensitivity may be underestimated because the denominator of the T/C value decreases. Case 14 had a low growth rate of 0.87 , suggesting the possibility of underestimating as reported. Therefore, further improvement of culture conditions is important to obtain accurate drug sensitivity test results.

A comparison of the clinical effect judgment of preoperative TPF therapy and IACRT which was treated with OSCC and the sensitivity test result of CD-DST revealed that the clinical correlation rate was $92.8 \%$ (13 of 14 cases) in combine with TPF therapy and IACRT, which reflected the clinical effect adequately. The clinical correlation rate of the CD-DST in OSCC was comparable to the previously reported clinical correlation rate of $91 \%$ for breast cancer, gastric cancer, and rectal cancer (6). From these results, CD-DST may predict the response of OSCC to multidrug combination therapy and the therapeutic effect IACRT.

In the future, cancer chemotherapy based on drug susceptibility test, including that for OSCC, could enable appropriate selection of patients, as well as treatment regimens that emphasize function preservation; improve quality of life; and provide treatment options for unresectable and refractory cases. However, Suzuki et al (30) reported differences in anticancer drug sensitivity testing between primary and metastatic OSCC tumors and suggested repeat chemotherapy drug sensitivity testing for metastatic/recurrent cases. The timing and performance of drug susceptibility test for recurrent/metastatic OSCC tumors need investigation in the future. In addition, accumulation and analysis of additional cases are necessary to verify our results on CD-DST in OSCC patients. Further study on the correlation between clinical effect and survival rate should be carried out to determine the range of clinical application of the CD-DST in OSCC.

In conclusion, CD-DST data of TPF and IACRT obtained by biopsy or surgical-fresh specimens of OSCC correlated to the disease control effects of chemotherapy for it. To select the chemotherapy regimen and to expect a better response from each patient with OSCC, the clinical application of this in vitro sensitivity test should be prospectively driven under clinical study using more OSCC patients.

\section{References}

1. Umeda M, Komatsubara H, Ojima Y, Minamikawa T, Shigeta T, Shibuya Y, Yokoo S and Komori T: Lack of survival advantage in patients with advanced, resectable squamous cell carcinoma of the oral cavity receiving induction chemotherapy with cisplatin (CDDP), docetaxel (TXT) and 5-fluorouracil (5FU). Kobe J Med Sci 50: 189-196, 2004

2. Katori H, Tsukuda M, Mochimatu I, Ishitoya J, Kawai S, Mikami Y, Matsuda H, Tanigaki Y, Horiuchi C, Ikeda Y, et al: Phase I trial of concurrent chemoradiotherapy with docetaxel, cisplatin and 5-fluorouracil (TPF) in patients with locally advanced squamous cell carcinoma of the head and neck (SCCHN). Br J Cancer 90: 348-352, 2004.

3. Ahn JS, Cho SH, Kim OK, Lee JK, Yang DH, Kim YK, Lee JJ, Lim SC, Kim HJ, Chung WK and Chung IJ: The efficacy of an induction chemotherapy combination with docetaxel, cisplatin and 5-FU followed by concurrent chemoradiotherapy in advanced head and neck cancer. Cancer Res Treat 39: 93-98, 2007.

4. Fuwa N, Ito Y, Matsumoto A, Kamata M, Kodaira T, Furutani K, Sasaoka M, Kimura Y and Morita K: A combination therapy of continuous superselective intraarterial carboplatin infusion and radiation therapy for locally advanced head and neck carcinoma. Phase I study. Cancer 89: 2099-2105, 2000

5. Kobayashi H, Tanisaka K, Kondo N, Mito Y, Koezuka M, Yokouchi H, Higashiyama M, Kodama K, Doi O, Yamada M, et al: Development of new in vitro chemosensitivity test using collagen gel droplet embedded culture and its clinical usefulness. Gan To Kagaku Ryoho 22: 1933-1939, 1995 (In Japanese).

6. Kobayashi H, Tanisaka K, Doi O, Kodama K, Higashiyama M, Nakagawa H, Miyake M, Taki T, Hara S, Yasutomi M, et al: An in vitro chemosensitivity test for solid human tumors using collagen gel droplet embedded cultures. Int J Oncol 11: 449-455, 1997.

7. Naitoh H, Yamamoto H, Murata S, Kobayashi H, Inoue K and Tani T: Stratified phase II trial to establish the usefulness of the collagen gel droplet embedded culture-drug sensitivity test (CD-DST) for advanced gastric cancer. Gastric Cancer 17: 630-637, 2014.

8. Ochiai T, Nishimura K, Watanabe T, Kitajima M, Nakatani A, Inou T, Washio M, Sakuyama N, Sato T, Kishine K, et al: Individualized chemotherapy for colorectal cancer based on the collagen gel droplet-embedded drug sensitivity test. Oncol Lett 4: 621-624, 2012.

9. Mekata E, Sonoda H, Shimizu T, Tatsuta T, Yamaguchi T, Endo Y and Tani T: Clinical predictive value of in vitro anticancer drug sensitivity test for the therapeutic effect of adjuvant chemotherapy in patients with stage II-III colorectal cancer. Mol Clin Oncol 1: 763-767, 2013.

10. Sakuma K, Tanaka A and Mataga I: Collagen gel droplet-embedded culture drug sensitivity testing in squamous cell carcinoma cell lines derived from human oral cancers: Optimal contact concentrations of cisplatin and fluorouracil. Oncol Lett 12: 4643-4650, 2016.

11. Takamura Y, Kobayashi H, Taguchi T, Motomura K, Inaji H and Noguchi S: Prediction of chemotherapeutic response by collagen gel droplet embedded culture-drug sensitivity test in human breast cancers. Int J Cancer 98: 450-455, 2002.

12. Eisenhauer EA, Therasse P, Bogaerts J, Schwartz LH, Sargent D, Ford R, Dancey J, Arbuck S, Gwyther S, Mooney M, et al: New response evaluation criteria in solid tumours: Revised RECIST guideline (version 1.1). Eur J Cancer 45: 228-247, 2009.

13. Inuyama $Y$ and Takeda C: A cooperative phase II study of cisplatin in patients with head and neck cancer. Gan To Kagaku Ryoho 13: 232-238, 1986 (In Japanese).

14. Jacobs C, Lyman G, Velez-García E, Sridhar KS, Knight W, Hochster H, Goodnough LT, Mortimer JE, Einhorn LH, Schacter L, et al: A phase III randomized study comparing cisplatin and fluorouracil as single agents and in combination for advanced squamous cell carcinoma of the head and neck. J Clin Oncol 10: 257-263, 1992.

15. Inuyama Y, Kataura A, Togawa K, Saijo S, Satake B, Takeoda S, Konno A, Ebihara S, Sasaki Y, Kida A, et al: Late phase II clinical study of RP56976 (docetaxel) in patients with advanced/ recurrent head and neck cancer. Gan To Kagaku Ryoho 26: 107-116, 1999 (In Japanese).

16. Zhong LP, Zhang CP, Ren GX, Guo W, William WN Jr, Sun J, Zhu HG, Tu WY, Li J, Cai Y, et al: Randomized phase III trial of induction chemotherapy with docetaxel, cisplatin and fluorouracil followed by surgery versus up-front surgery in locally advanced resectable oral squamous cell carcinoma. J Clin Oncol 31: 744-751, 2013. 
17. Vermorken JB, Mesia R, Rivera F, Remenar E, Kawecki A Rottey S, Erfan J, Zabolotnyy D, Kienzer HR, Cupissol D, et al: Platinum-based chemotherapy plus cetuximab in head and neck cancer. N Engl J Med 359: 1116-1127, 2008.

18. Kiyota N, Tahara M, Kadowaki S, Fuse N, Doi T, Minami H and Ohtsu A: Systemic chemotherapy with cisplatin plus 5-FU (PF) for recurrent or metastatic squamous cell carcinoma of the head and neck (R/M SCCHN): Efficacy and safety of a lower dose of $\mathrm{PF}(80 / 800)$ at a single institution in Japan. Jpn J Clin Oncol 39: 225-230, 2009.

19. Robbins KT, Kumar P, Harris J, McCulloch T, Cmelak A, Sofferman R, Levine P, Weisman R, Wilson W, Weymuller E and Fu K: Supradose intra-arterial cisplatin and concurrent radiation therapy for the treatment of stage IV head and neck squamous cell carcinoma is feasible and efficacious in a multi-institutional setting: Results of Radiation Therapy Oncology Group Trial 9615. J Clin Oncol 23: 1447-1454, 2005.

20. Robbins KT, Kumar P, Regine WF, Wong FS, Weir AB III, Flick P, Kun LE, Palmer R, Murry T, Fontanesi J, et al: Efficacy of targeted supradose cisplatin and concomitant radiation therapy for advanced head and neck cancer: The memphis experience. Int J Radiat Oncol Biol Phys 38: 263-271, 1997.

21. Vermorken JB, Remenar E, van Herpen C, Gorlia T, Mesia R, Degardin M, Stewart JS, Jelic S, Betka J, Preiss JH, et al: Cisplatin, fluorouracil, and docetaxel in unresectable head and neck cancer. N Engl J Med 357: 1695-1704, 2007.

22. Posner MR, Hershock DM, Blajman CR, Mickiewicz E, Winquist E, Gorbounova V, Tjulandin S, Shin DM, Cullen K Ervin TJ, et al: Cisplatin and fluorouracil alone or with docetaxel in head and neck cancer. N Engl J Med 357: 1705-1715, 2007.
23. Salmon SE, Hamburger AW, Soehnlen B, Durie BG, Alberts DS and Moon TE: Quantitation of differential sensitivity of human-tumor stem cells to anticancer drugs. N Engl J Med 298: 1321-1327, 1978

24. Kondo T, lmamura $\mathrm{T}$ and lchibashi $\mathrm{H}$ : In vitro test for sensitivity of tumor to carcinostatic agents. Gan 57: 113-121, 1966.

25. Inaba M, Tashiro T, Sato S, Ohnishi Y, Tanisaka K, Kobayashi H and Koezuka M: In vitro-in vivo correlation in anticancer drug sensitivity test AUC-based concentrations and collagen gel droplet embedded culture. Oncology 53: 250-257, 1996.

26. Araki Y, Isomoto H, Matsumoto A, Kaibara A, Yasunaga M, Hayashi K, Yatsugi $\mathrm{H}$ and Yamauchi K: An in vitro chemosensitivity test for colorectal cancer using collagen-gel droplet embedded cultures. Kurume Med J 46: 163-166, 1999.

27. Kawamura $\mathrm{M}$, Inoue $\mathrm{Y}$, Oyama $\mathrm{T}$ and Kobayashi $\mathrm{K}$ : Chemosensitivity test for uresectable non-small cell lung cancer. Nihon Geka Gakkai Zasshi 103: 229-232, 2002 (In Japanese).

28. Yamamoto Y, Watanabe Y, Ishida N, Hidaka T, Sugishita H, Yoshida M, Yukumi S, Sato K, Nakagawa H, Horiuchi A and Kawachi K: Collagen gel droplet-embedded culture drug sensitivity test in human breast cancer. Gan To Kagaku Ryoho 35: 793-796, 2008.

29. Nakagawa T, Takahashi H, Kamiyama T, Nakanishi $K$, Takahashi M, Watanabe K, Taguchi K, Tsukahara M, Nakajima H, Kamachi $\mathrm{H}$, et al: In vitro chemosensitivity test for hepatocellular carcinoma using collagen-gel droplet embedded cultures. Gan To Kagaku Ryoho 31: 2145-2149, 2004 (In Japanese).

30. Suzuki M, Ishikawa H, Tanaka A and Mataga I: Heterogeneity of anticancer drug sensitivity in squamous cell carcinoma of the tongue. Hum Cell 24: 21-29, 2011. 